\title{
Patterns and predictors of COVID-19 occurrence and severity in vaccinated priority groups
}

\section{Upinder Kaur}

Banaras Hindu University

\section{Sapna Bala}

Banaras HIndu University

Bisweswar Ojha

Banaras Hindu University

\section{Bhairav Kumar Pathak}

Banaras HIndu University

Aditi Joshi

Banaras Hindu University

Ashish Kumar Yadav

Banaras Hindu University

\section{Anup Singh}

Banaras Hindu University

\section{Sangeeta Kansal}

Banaras Hindu University

Sankha Shubhra Chakrabarti ( $\sim$ sankha.geriatrics@gmailc.om )

Banaras Hindu University

\section{Research Article}

Keywords: COVID-19, severity, sex-wise dimorphism, asthma, lung disease, obesity, vaccination dosing schedule

Posted Date: December 28th, 2021

DOI: https://doi.org/10.21203/rs.3.rs-1204671/v1

License: (c) (1) This work is licensed under a Creative Commons Attribution 4.0 International License.

Read Full License 


\section{Abstract}

Background: Varying protection rates have been observed with approved COVID-19 vaccines postapproval, in various real-world studies. However, little data exists on the clinical presentation and risk factors of occurrence and severity of COVID-19 in vaccinated individuals. The aim of the present study is to describe the patterns of presentation of COVID-19 in vaccinated individuals, and to determine the predictors of occurrence of COVID-19 and the predictors of severity in the vaccinated.

Methods: The study is a part of a prospective observational study ongoing since February 2021 in a tertiary teaching and research hospital of northern India. Individuals recruited in this study belong to a high-risk group comprising health care workers and elderly. COVISHIELD, based on ChAdOx1 nCoV-19 platform was the vaccine received by the participants based on policy on allocation.

Primary outcomes of the study are the short term and long-term adverse events following immunization (AEFI) with COVID-19 vaccines. Secondary outcomes include the rates of occurrence of COVID-19 and severity of COVID-19. Data on these have been already reported. The clinical presentation, typical and atypical manifestations, time to symptomatic recovery and patterns of post-COVID-19 complaints were analysed in the current study. Logistic regression analysis was performed to predict the risk factors of occurrence of COVID-19 in the vaccinated and the determinants of severe forms of the disease in this group.

Findings: 1500 individuals completed at least two month follow up. Of these, 418 developed laboratory confirmed or suspected COVID-19. Fever was the commonest symptom (72\%), followed by features of rhinitis $(41 \%)$ and cough (34\%). Nearly $12 \%$ individuals had only one symptom or none. Cardiovascular involvement was seen in more than $2 \%$ of affected. Time to symptomatic recovery varied from 1-75 days and $11 \%$ had post COVID-19 complaints at two month follow up, most common being generalized weakness. Results of regression analysis showed 1.6 times higher odds of contracting the disease in females and young individuals $<40$ years of age $(P<0.001)$. Overweight individuals and those receiving only one dose were at 1.4 times and 3 times higher odds of contracting COVID-19 compared to those with normal body mass index and those who were fully vaccinated. Individuals receiving two doses at a gap of $<30$ days were at 7 times higher odds of disease acquisition compared to those receiving the second dose at an extended gap of $>60$ days $(P=0.01)$.

With respect to severity, males and those receiving only one dose each had 3 times higher odds of suffering from moderate to severe COVID-19 compared to females and fully vaccinated. Persons with preexisting lung disease, such as asthma had 6 times higher odds of suffering from moderate to severe COVID-19 $(P=0.024)$. No association with the occurrence or severity of disease was observed with any other co-morbidity or the use of renin-angiotensin-aldosterone system (RAAS) blockers.

Interpretation: Sex wise differences exist with respect to occurrence and severity of COVID-19. Two doses of vaccine, compared to one dose provide considerable protection against occurrence as well as severity. Full vaccination with extended dosing interval should be the optimal strategy and should be carried out 
when the community burden of cases is negligible. Future studies are needed to explore the sex wise differences in the propensity and severity of COVID-19. Further, the risk association of asthma phenotypes with COVID-19 needs to be investigated.

Funding: The study received no funding support

\section{Introduction}

The ChAdOx1 nCoV-19 vaccine, based on recombinant chimpanzee adenovirus platform and the mRNAbased vaccines of Pfizer and Moderna have been administered in the largest number of countries globally. COVISHIELD manufactured by Serum Institute of India is based on the ChAdOx1 platform and has been the major candidate vaccine in the mass roll out programme in India. Short term safety profile of ChAdOX1 nCoV19 was observed to be favourable, and efficacy close to 70\% against COVID-19 was demonstrated in controlled settings. Post approval, modest reduction in the effectiveness of COVID-19 vaccines has been observed in the real-world. ${ }^{1,2}$ We recently reported a close to $25 \%$ rate of SARS-CoV-2 breakthrough infections in high-risk priority groups. ${ }^{3}$ However, studies which provide detailed clinical description of occurrence of COVID-19 in vaccinated individuals, as well as the potential risk factors of disease occurrence and severity in them are scarce. To contribute towards this missing data, we decided to analyse the findings of an ongoing prospective observational study on long term safety of COVISHIELD whose primary and secondary outcome measures have already been reported preliminarily by us. ${ }^{3,4}$ Our aim was to provide a detailed description of the clinical patterns of COVID-19, the typical and atypical manifestations of disease, management, outcomes, and incidence as well as patterns of post-COVID-19 complaints in vaccinated individuals. More importantly, for the first time, we provide an analysis of the risk factors of occurrence and severity of COVID-19 in vaccinated individuals.

\section{Materials And Methods}

\subsection{Study design and setting}

Ours is a prospective observational study which started in February 2021 in a tertiary teaching and research hospital of north India with the primary objective of assessing short-term and long-term safety of COVID-19 vaccines and the secondary objective of estimating the occurrence of COVID-19 in priority groups. Vaccinees were enrolled from the study hospital and two associated centres. ${ }^{4}$ COVISHIELD was the vaccine allotted to the centres as per government policies. The authors UK and SSC had access to the entire data.

\subsection{Study participants:}

All the individuals who received vaccines, in the three centres, and who provided consent to participate were enrolled in the study till attainment of sample size. As per national policy for prioritisation of vaccine recipients, the enrolled vaccinees were health care workers in the initial phase of study, with subsequent 
enrolment of elderly in the later phase. The participants are being contacted telephonically at specific time intervals till one year follow up of each participant is complete. Details of design are already published. ${ }^{4}$

\subsection{Outcomes measured:}

Primary safety outcomes were recently published by our group. ${ }^{4}$ For secondary outcome which was the occurrence of post-vaccination COVID-19, the participants were enquired about the occurrence of symptoms related to COVID-19. COVID-19 cases were classified as 'confirm' and 'suspect' as defined previously. ${ }^{3}$ The classification was based on guidance from the Ministry of Health \& Family Welfare (MoHFW), Government of India recommendations on COVID-19. Here we perform analysis of risk factors of disease occurrence post-vaccination and determinants of severity post-vaccination. For simplification of analysis, both 'confirm' and 'suspect' cases are clubbed into group A and the remaining are classified as group B. MoHFW guidelines were used to categorize the severity of disease. Individuals with asymptomatic or mild disease were incorporated in one group and were compared to individuals developing moderate or severe COVID-19. Potential risk factors analysed were demographic variables, the presence of co-morbidities, use of RAAS (renin angiotensin aldosterone system) blockers, doses of vaccine received and the interval between the two doses. Incidence and patterns of post COVID-19 symptoms if any, persisting at two months follow up, were also assessed. In case of death of any participant, necessary details were obtained from the caregivers and proof of cause of death certification was sought. ${ }^{3}$

\subsection{Ethical permission}

The study started after obtaining permission from the Ethics Committee of the Institute of Medical Sciences, Banaras Hindu University, and written informed consent was taken from all the participants.

\subsection{Data sources/measurement}

Data pertaining to demography, medical history including history of prior SARS-CoV-2 positivity, existing co-morbidities, vaccine details, and concurrent drug history was recorded in a pre-designed case report form. Data concerning post-vaccination COVID-19 was entered as per pre-designed format electronically. Detailed methodology has been described in earlier publication. ${ }^{4}$

\subsection{Sample size}

The calculation of sample size for the main study has already been described by us in an earlier paper. ${ }^{4}$

\subsection{Statistical analysis:}

Results were recorded as frequencies as well as percentages for data pertaining to demographics and to patterns of COVID-19 symptoms developing in vaccinated individuals. Values are presented as mean and median for non-skewed and skewed variables respectively. To find association between various tentative 
risk factors and development of COVID-19, Chi square test was applied for dichotomous variables such as gender, presence of co-morbidities, number of doses of vaccine, previous history of COVID-19 (confirm/suspect) and for categorized quantitative variables such as body mass index (BMI) and age. Variables with $\mathrm{P}<0.05$ on bivariate analysis or those deemed to be clinically relevant were incorporated in the final regression model. Corroboration was done with multivariable fractional polynomial (MFP) method using MFP package from STATA 16. Binomial logistic regression analysis was conducted first, to determine the predictors of occurrence of COVID-19 and second, to determine the risk factors of moderate-severe COVID-19. To determine the effect of dosing interval of vaccines, regression analysis was done separately after excluding those who received only one dose and those who developed COVID19 between the two doses of vaccine. In case of significant correlation ( $r>0.7)$ between independent variables, variance inflation factor was used to detect multi collinearity and a cut off value of 2.5 was taken to exclude the collinearity.

\subsection{Role of funding source}

This study had no funding support.

\section{Results}

Of 1500 vaccinated individuals completing two months of successful follow up, 418 were diagnosed as cases of COVID-19 ('confirm' or 'suspect') at some point during their follow-up. They were classified in group A. The remaining 1082 were clubbed in group B. Table 1 shows details pertaining to demographic parameters and co-morbidities in the two groups. Further, Table 1 mentions the detailed clinical features, treatment received, time to symptomatic recovery and post-COVID-19 complaints of those diagnosed with COVID-19 (group A). Median number of symptoms was 3 (Q1, Q3 2, 4). Fever, rhinitis, cough, and sore throat were the common symptoms seen respectively in $71 \%, 41 \%, 34 \%$ and $26 \%$ participants.

Cardiovascular involvement was seen in $2.4 \%$ of affected. Around $7 \%$ of affected had only one symptom of which fever, sore throat, and cough, were seen in $32 \%, 18 \%$ and $14 \%$ individuals respectively. Time to symptomatic recovery varied from $1-75$ days and around $11 \%$ of affected had post COVID-19 complaints at two month follow up. Generalized weakness was the commonest post-COVID-19 complaint seen in $44.4 \%$ of those with post-COVID-19 complaints. This was followed by persistence of cough $(17.8 \%)$ and dyspnoea (13.3\%). In univariate analysis, age, gender, and number of doses of vaccine received shared statistically significant association with the occurrence of disease. Those with $P$ value $<0.05$ and those deemed clinically important were analysed in logistic regression analysis.

Younger individuals ( $<40$ years), females, overweight individuals and those who received only one dose of vaccine had respectively 1.7-, 1.7-, 1.4- and 2.9-times higher odds of contracting COVID-19, with statistical significance. Dosing interval was also a strong determinant of disease occurrence. The odds of contracting COVID-19 was 6.7 times in those receiving the two doses at $\leq 30$ days interval compared to those who received the second dose at a gap of $>60$ days. Compared to the group receiving the second dose at 31-60 days interval, the odds of contracting COVID-19 was 1.6 times higher in those who received 
the second dose at a gap of $\leq 30$ days from first dose. Both risk associations were statistically significant.

Gender, pre-existing lung disease, use of RAAS blockers and number of doses of vaccine received shared a statistically significant association with disease severity. Percentage of individuals with pre-existing lung disease was more than three times higher in the group with moderate-severe form of COVID-19 versus the group with asymptomatic-mild COVID-19. Likewise, the usage of RAAS blockers was two times more prevalent in individuals with moderate-severe COVID-19. To determine the effect of dosing interval on severity of disease, logistic regression was performed on $n=382$, after excluding those who received only one dose and those who developed COVID between the two doses. Males, recipients of only one dose and those with pre-existing lung disease had respectively 3.6 times, 3 times and nearly 6 times higher odds of developing moderate-severe form of disease compared to females, those who received two doses and those without any pre-existing lung disease. The risk association was statistically significant. Though higher odds of moderate-severe form of disease was seen in the group receiving the second dose of vaccine at $\leq 30$-day interval compared to those at $>30$ days, association was not statistically significant. The association of RAAS blockers with severity of disease was nullified in regression analysis.

\section{Discussion}

Compared to findings in controlled settings, modest reduction has been observed in vaccine effectiveness in real world situations, with protection rates against COVID-19 varying from $24-65 \%$. ${ }^{1,2}$ The percentage of vaccinees developing symptomatic COVID-19 was low (<1\%) in randomized controlled trials of ChAdOx1 nCoV-19. In the real-world settings, however, high SARS-CoV-2 infection and disease rates have been observed in vaccinated individuals. In our previously published study, lab confirmed COVID-19 was seen in $41 \%$ individuals who received only one dose and around $20 \%$ individuals receiving both doses of the vaccine. Corresponding rates were around $67 \%$ and $35 \%$ in subgroup of 'doctors' recruited in the study. Most of the infections in our study were observed to be breakthrough and were mild in severity. ${ }^{3}$ Here we have analysed the spectrum of symptoms in COVID-19 in vaccinated individuals, the risk factors of breakthrough infections, and the risk factors of moderate to severe disease in vaccinated individuals.

The commonly occurring symptoms in vaccinated individuals in this study were fever (72\%), followed by features of rhinitis (41\%) and cough (34\%). Around $5 \%$ of individuals with lab confirmed COVID-19 were completely asymptomatic and around $7 \%$ had only one symptom. Cardiovascular involvement in the form of significant changes in blood pressure was observed in $2.4 \%$ and majority of these patients reported an elevation of blood pressure during the illness. Atypical organ involvement in the form of ocular symptoms was observed in more than $2 \%$ of affected, emphasizing the need to be vigilant against atypical symptoms and signs in the face of changing variants of SARS-CoV-2. Around $11 \%$ had some post COVID-19 complaint at two months follow up, most common being generalized weakness. This attains special significance in future deliberations of how vaccines may have any role of importance in long COVID. Post-vaccination COVID-19 symptomatology has not been elaborated widely. In a 
metanalysis conducted in the pre-vaccination period, prevalence of COVID-19 in health care workers has varied from 0.4 to $57 \%$ with females constituting the majority. Fever, anosmia, and myalgia were the commonest symptoms. ${ }^{5}$

In the current study, on logistic regression analysis, occurrence of COVID-19 was statistically more common in females (OR 1.6), in the younger age group (OR 1.6), in individuals who were overweight (OR 1.4) and in those who received only one dose of the vaccine (OR 2.9). The risk association of COVID-19 with age, gender and BMI was corroborated when regression analysis was performed on individuals receiving two doses of the vaccine. High BMI, previously, has been associated with severe form of COVID19. 6,7 While males and females are believed to have a similar susceptibility to the acquisition of the disease, some studies have shown a higher incidence rate of COVID-19 in females, particularly among health care workers. ${ }^{8}$ Geographical variations also exist with respect to gender and susceptibility to COVID-19. In certain regions of Peru for example, the incidence rate of COVID-19 was shown to be higher in females than males. ${ }^{9}$ Interestingly, females are also at higher risk of getting adverse events following immunization with COVID-19 vaccines. ${ }^{4,10,11}$ No difference in the occurrence of COVID-19 was seen with respect to other variables such as pre-vaccination history of COVID-19, medical co-morbidities and with the use of RAAS blockers. Since the emergence of COVID-19, the members of RAAS blocker class were hypothesized to enhance the propensity towards SARS-CoV-2 infection as well as severe COVID-19 disease. However, the association of these drugs with disease severity was refuted in some of the largest meta-analyses conducted by us and others. ${ }^{12,13}$ The absence of a relation between COVID-19 occurrence in vaccinated people and pre-vaccination history of prior infection may imply that boosting effect of vaccines on naturally acquired immunity needs to be explored further.

Dosing interval was observed to be a significant influencer of occurrence of COVID-19 in this study. More than six times (OR 6.7) and nearly 1.6 times higher risk of contracting the disease was observed in individuals receiving two doses of vaccine at $\leq 30$ days interval compared to those who received the second dose at gap of more than 60 days and at 31-60 days, respectively. The corresponding risk was more than four times (OR 4.13) in those receiving second dose of vaccine at 31-60 days compared to those who got the second dose at an interval exceeding 60 days with marginal statistical significance ( $P$ 0.059). Overall, better protection rates have been demonstrated with increase in the dosing interval of ChAdOx $1 \mathrm{nCoV}-19$ vaccine. This was one of the reasons provided by various drug regulatory authorities, for increasing the dosing interval of ChAdOx $1 \mathrm{nCoV}-19$ from 28 days to 84 days. ${ }^{14}$

With respect to severity of COVID-19, statistically significant association was seen with gender, preexisting lung disease, use of RAAS blockers and number of doses of vaccine. Except the use of RAAS blockers, the association of remaining three factors remained statistically significant on logistic regression analysis too. Males and those receiving only one dose of the vaccine had close to 3 times higher odds of developing moderate-severe form of the disease compared to females and those receiving both doses. With marginal statistical significance $(p=0.08)$, individuals with pre-existing lung disease were at more than three times risk of developing moderate to severe COVID-19 compared to those with no 
pre-existing lung illness. The risk association of severity of COVID-19 was enhanced in the regression analysis confined to individuals receiving two doses of vaccine $(n=382)$ with an odds ratio for male gender of 3.6 and OR of 5.9 for pre-existing lung disease. Males have been found to be at higher risk of severe form of COVID-19 in several other studies done in the pre-vaccination period. ${ }^{6,7}$ Increased COVID19 severity in males might be explained to some extent by androgen facilitated SARS-CoV-2 entry or immune regulation by male hormones. Testosterone can positively up regulate the expression of ACE-2 and possibly of TMPRSS2, both of which are involved in SARS-CoV-2 S protein entry and infectivity. ${ }^{15}$ Testosterone is also known to blunt the protective functions of neutrophils, macrophages, and dendritic cells against the invading pathogen. ${ }^{16}$ Asthma was the predominant pre-existing lung disease in our vaccinated cohort. Evidence surrounding association of asthma with COVID-19 morbidity and mortality is conflicting. While CDC suggests moderate-severe asthma to be a potential risk factor of severe form of COVID-19, no significant association was claimed between asthma and disease severity by others. ${ }^{17}$ Severity of COVID-19 may also be governed by the Th phenotype of asthma as has been suggested by some. ${ }^{17,18}$

Though a numerically higher percentage of individuals above 40 years of age, overweight individuals, those with past history of COVID-19 and those with co-morbidities was seen in the group developing the severe forms of disease, the association of these factors was not statistically significant with disease severity. Old age, obesity, diabetes mellitus, hypertension, kidney disease and immunosuppression are some of the predictors of worse COVID-19 outcomes in earlier studies. ${ }^{19}$ Absence of association of COVID-19 severity with these factors in our study should be taken with caution as the population chosen was predominantly composed of the young and percentage of those with co-morbidities was low. The findings, hence, may not be extrapolated to the general population. Dosing interval was not a statistically significant predictor of severity of disease.

\section{Limitations}

The present study was designed for vaccinated individuals only and comment on effectiveness could not be made at this point. Another study involving unvaccinated individuals has been started by the authors and the results shall be available by early 2022. Being a telephonic survey-based study, the possibility of recall bias may exist, but the participants were mostly healthcare and other frontline workers and the data provided by them can be considered mostly reliable. Finally, the study sample is not representative of the general Indian population, and hence findings may not be generalized to all.

\section{Conclusion}

The current study is one of the few to explore the characteristics and risk factors of post-vaccination COVID-19. Among the vaccinated, younger individuals and females have 1.6 times higher risk of acquisition of COVID-19. Being overweight, also enhances the risk of contracting the disease by around 1.4 times. With respect to severity, males have more than 3 times higher risk of suffering from severe 
forms of disease. Presence of pre-existing lung disease such as asthma was seen to enhance the risk of developing moderate to severe COVID-19 by six times. Fully vaccinated individuals were at three times lower risk of contracting COVID-19 as well as developing moderate to severe disease. Since no difference was observed between occurrence and severity of COVID-19 in vaccinated individuals with or without prior SARS-CoV-2 infection, further real-world studies of boosting effect of vaccination on naturally acquired immunity need to be performed. Dosing interval was found to be a strong determinant of acquisition of disease. Nearly seven times lower risk of occurrence of COVID-19 was seen in individuals receiving the second dose at extended interval of more than 60 days. Thus, while full vaccination is an important strategy in the fight against the pandemic, planning on the optimal timing of the dosing schedule is equally relevant. Vaccinating the individuals with cases still prevalent in the community can transform the vaccination centres to infection spreading sites. Optimal timing should be when community burden of disease is close to negligible. Further focused studies are needed to investigate the sex-wise differences in disease acquisition and severity and the risk association of COVID-19 with asthma phenotypes.

\section{Declarations}

\section{Acknowledgement: None}

\section{Conflict of Interest: None}

Ethical Approval: The study was approved by the ethics committee of the Institute of Medical Sciences, Banaras Hindu University. No human experimentation was performed. All protocols were performed as per the Declaration of Helsinki and its later modifications. Written informed consent was obtained from every participant enrolled.

\section{References}

1. Satwik R, Satwik A, Katoch S, Saluja S. ChAdOx1 nCoV-19 effectiveness during an unprecedented surge in SARS COV-2 infections. Eur J Intern Med 2021; 93: 112-3.

2. Victor PJ, Mathews KP, Paul H, Mammen JJ, Murugesan M. Protective Effect of COVID-19 Vaccine Among Health Care Workers During the Second Wave of the Pandemic in India. Mayo Clin Proc 2021; 96: 2493-4.

3. Kaur U, Bala S, Ojha B, Jaiswal S, Kansal S, Chakrabarti SS. Occurrence of COVID-19 in priority groups receiving ChAdOx $1 \mathrm{nCoV}-19$ coronavirus vaccine (recombinant): A preliminary analysis from north India. J Med Virol 2022; 94: 407-12.

4. Kaur U, Ojha B, Pathak BK, et al. A prospective observational safety study on ChAdOx1 nCoV-19 corona virus vaccine (recombinant) use in healthcare workers- first results from India. EClinicalMedicine 2021; 38: 101038. 
5. Gómez-Ochoa SA, Franco OH, Rojas LZ, et al. COVID-19 in Health-Care Workers: A Living Systematic Review and Meta-Analysis of Prevalence, Risk Factors, Clinical Characteristics, and Outcomes. Am J Epidemiol 2021; 190: 161-75.

6. Li X, Xu S, Yu M, et al. Risk factors for severity and mortality in adult COVID-19 inpatients in Wuhan. J Allergy Clin Immunol 2020; 146: 110-8.

7. Palaiodimos L, Kokkinidis DG, Li W, et al. Severe obesity, increasing age and male sex are independently associated with worse in-hospital outcomes, and higher in-hospital mortality, in a cohort of patients with COVID-19 in the Bronx, New York. Metabolism 2020; 108: 154262.

8. O'Brien J, Du KY, Peng C. Incidence, clinical features, and outcomes of COVID-19 in Canada: impact of sex and age. J Ovarian Res 2020; 13: 137.

9. Ramírez-Soto MC, Arroyo-Hernández H, Ortega-Cáceres G. Sex differences in the incidence, mortality, and fatality of COVID-19 in Peru. PLoS One 2021; 16: e0253193.

10. Konu YR, Gbeasor-Komlanvi FA, Yerima M, et al. Prevalence of severe adverse events among health professionals after receiving the first dose of the ChAdOx1 nCoV-19 coronavirus vaccine (Covishield) in Togo, March 2021. Arch Public Health 2021; 79: 207.

11. Joshi RK, Muralidharan CG, Gulati DS, et al. Higher incidence of reported adverse events following immunisation (AEFI) after first dose of COVID-19 vaccine among previously infected health care workers. Med journal, Armed Forces India 2021; 77: S505-7.

12. Kaur U, Chakrabarti SS, Patel TK. Renin-angiotensin-aldosterone system blockers and region-specific variations in COVID-19 outcomes: findings from a systematic review and meta-analysis. Ther Adv drug Saf 2021; 12: 20420986211011344.

13. Xu J, Teng Y, Shang L, et al. The Effect of Prior Angiotensin-Converting Enzyme Inhibitor and Angiotensin Receptor Blocker Treatment on Coronavirus Disease 2019 (COVID-19) Susceptibility and Outcome: A Systematic Review and Meta-analysis. Clin Infect Dis 2021; 72: e901-13.

14. Voysey M, Costa Clemens SA, Madhi SA, et al. Single-dose administration and the influence of the timing of the booster dose on immunogenicity and efficacy of ChAdOx 1 nCoV-19 (AZD1222) vaccine: a pooled analysis of four randomised trials. Lancet (London, England) 2021; 397: 881-91.

15. Baratchian M, McManus JM, Berk MP, et al. Androgen regulation of pulmonary AR, TMPRSS2 and ACE2 with implications for sex-discordant COVID-19 outcomes. Sci Rep 2021; 11: 11130.

16. Ben-Batalla I, Vargas-Delgado ME, von Amsberg G, Janning M, Loges S. Influence of Androgens on Immunity to Self and Foreign: Effects on Immunity and Cancer. Front Immuno/ 2020; 11: 1184.

17. Muñoz X, Pilia F, Ojanguren I, Romero-Mesones C, Cruz M-J. Is asthma a risk factor for COVID-19? Are phenotypes important? ERJ Open Res 2021; 7: 00216-2020.

18. Fernando M, Agusti A, Dharmage S, Lodge $C$. Are women with asthma at increased risk for severe COVID-19? Lancet Respir Med 2021; 9: 125-6.

19. Gallo Marin B, Aghagoli G, Lavine K, et al. Predictors of COVID-19 severity: A literature review. Rev Med Virol 2021; 31: 1-10. 


\section{Table 1}

Table 1 is available in the Supplementary Files section.

\section{Supplementary Files}

This is a list of supplementary files associated with this preprint. Click to download.

- Table1.pdf 